\title{
Gröbner-Shirshov Bases for Temperley-Lieb Algebras of Complex Reflection Groups
}

\author{
Jeong-Yup Lee ${ }^{1} \mathbb{D}$, Dong-il Lee ${ }^{2, *} \mathbb{C}$ and SungSoon $\mathrm{Kim}^{3}$ \\ 1 Department of Mathematics Education, Catholic Kwandong University, Gangwondo 25601, Korea; \\ jylee@cku.ac.kr \\ 2 Department of Mathematics, Seoul Women's University, Seoul 01797, Korea \\ 3 LAMFA-CNRS UMR 7352, Université de Picardie JV-Mathématiques, 80039 Amiens, France, \\ (membre assoc. équipe des groupes, IMJ-PRG Univ. Paris 7); sungsoon.kim@u-picardie.fr \\ * Correspondence: dilee@swu.ac.kr; Tel.: +82-2-970-5677
}

Received: 19 August 2018; Accepted: 21 September 2018; Published: 26 September 2018

\begin{abstract}
We construct a Gröbner-Shirshov basis of the Temperley-Lieb algebra $\mathcal{T}(d, n)$ of the complex reflection group $G(d, 1, n)$, inducing the standard monomials expressed by the generators $\left\{E_{i}\right\}$ of $\mathcal{T}(d, n)$. This result generalizes the one for the Coxeter group of type $B_{n}$ in the paper by Kim and Lee We also give a combinatorial interpretation of the standard monomials of $\mathcal{T}(d, n)$, relating to the fully commutative elements of the complex reflection group $G(d, 1, n)$. More generally, the Temperley-Lieb algebra $\mathcal{T}(d, r, n)$ of the complex reflection group $G(d, r, n)$ is defined and its dimension is computed.
\end{abstract}

Keywords: Temperley-Lieb algebra; Gröbner-Shirshov basis; Catalan number; fully commutative element

MSC: Primary 20F55; Secondary 05E15; 16Z05

\section{Introduction}

The Temperley-Lieb algebra appears originally in the context of statistical mechanics [1], and later its structure has been studied in connection with knot theory, where it is known to be a quotient of the Hecke algebra of type $A$ in [2].

Our approach to understanding the structure of Temperley-Lieb algebras is from the noncommutative Gröbner basis theory, or the Gröbner-Shirshov basis theory more precisely, which provides a powerful tool for understanding the structure of (non-)associative algebras and their representations, especially in computational aspects. With the ever-growing power of computers, it is now viewed as a universal engine behind algebraic or symbolic computation.

The main interest of the notion of Gröbner-Shirshov bases stems from Shirshov's Composition Lemma and his algorithm [3] for Lie algebras and independently from Buchberger's algorithm [4] of computing Gröbner bases for commutative algebras. In [5], Bokut applied Shirshov's method to associative algebras, and Bergman mentioned the diamond Lemma for ring theory [6]. The main idea of the Composition-Diamond Lemma is to establish an algorithm for constructing standard monomials of a quotient algebra by a two-sided ideal generated by a set of relations called a Gröbner-Shirshov basis. Our set of standard monomials in this algorithm is a minimal set of monomials which are indivisible by any leading monomial of the polynomials in the Gröbner-Shirshov basis. The details on the Gröbner-Shirshov basis theory are given in Section 2.

The Gröbner-Shirshov bases for Coxeter groups of classical and exceptional types were completely determined by Bokut, Lee et al. in [7-11]. The cases for Hecke algebras and Temperley-Lieb algebras of type $A$ as well as for Ariki-Koike algebras were calculated by Lee et al. in [12-14]. We can also find some interesting works on the theory of quasi-monomials [15] with algorithms related to the Gröbner-Shirshov basis theory. 
This paper consists of two principal parts as follows:

(1) In the first part of this paper, extending the result for type $B_{n}$ in [16], we construct a Gröbner-Shirshov basis for the Temperley-Lieb algebra $\mathcal{T}(d, n)$ of the complex reflection group of type $G(d, 1, n)$ and compute the dimension of $\mathcal{T}(d, n)$, by enumerating the standard monomials which are in bijection with the fully commutative elements.

The main theorem goes as follows:

Theorem 1 (Main Theorem 4). The algebra $\mathcal{T}(d, n)$ has a Gröbner-Shirshov basis $\widehat{R}_{\mathcal{T}(d, n)}$ with respect to the monomial order $<$ (i.e., degree-lexicographic order with $E_{0}<E_{1}<\cdots<E_{n-1}$ ):

$$
\begin{aligned}
& E_{0}^{d}-(d-1) \delta E_{0}, \\
& E_{i} E_{0}-E_{0} E_{i} \quad \text { for } 1<i \leq n-1, \\
& E_{0} E_{1} E_{0}^{k} E_{1}-(k+1) E_{0} E_{1} \quad \text { for } 1 \leq k<d \text {, } \\
& E_{1} E_{0}^{k} E_{1} E_{0}-(k+1) E_{1} E_{0} \quad \text { for } 1 \leq k<d, \\
& \widehat{R}_{\mathcal{T}(d, n)}: \quad E_{i, 1} E_{0}^{k} E^{1, j} E_{i}-E_{i-2,1} E_{0}^{k} E^{1, j} E_{i} \quad \text { for } i>j+1 \geq 1 \text {, } \\
& E_{i}^{2}-\delta E_{i} \quad \text { for } 1 \leq i \leq n-1, \\
& E_{i} E_{j}-E_{j} E_{i} \quad \text { for } i>j+1>1, \\
& E_{i, j} E_{i}-E_{i-2, j} E_{i} \quad \text { for } i>j>0 \text {, } \\
& E_{j} E_{i, j}-E_{j} E_{i, j+2} \quad \text { for } i>j>0 \text {. }
\end{aligned}
$$

The cardinality of the set of $\widehat{R}_{\mathcal{T}(d, n)}$-standard monomials is

$$
\operatorname{dim} \mathcal{T}(d, n)=(d-1)\left(\mathfrak{F}_{n, n-1}(d)-1\right)+d C_{n}
$$

where $\mathfrak{F}_{n, k}(x)=\sum_{s=0}^{k} C(n, s) x^{k-s}$ is the $(n, k)$ th Catalan triangle polynomial, introduced in ([17], Section 2.3).

We remark here that by specializing $d=2$, we recover the formula for the Temperley-Lieb algebra $\mathcal{T}\left(B_{n}\right)$ of the $B_{n}$, found by Stembridge in [18].

(2) In the second part of this paper, we try to understand some combinatorial aspects on the dimension of the Temperley-Lieb algebra $\mathcal{T}(d, n)$.

In $[19,20]$, Kleshchev and Ram constructed a class of representations called homogeneous representations of the KLR algebra (or a quiver Hecke algebra) and showed that the homogeneous representations can be parametrized by the set of fully commutative elements of the corresponding Coxeter group (for simply laced cases only). Fan [21] and Stembridge [22] studied the fully commutative elements for Coxeter groups and proved that these elements parametrize the bases of the corresponding Temperley-Lieb algebras.

Motivated by the bijective correspondence, Feinberg and Lee studied the fully commutative elements of the Coxeter groups of types $A$ and $D$ in their papers $[23,24]$ and obtained a dimension formula of the homogeneous representations by using Dyck paths.

Their combinatorial strategy is as follows:

For type $A$, we decompose the fully commutative elements into natural subsets according to the lengths of fully commutative elements and show that the fully commutative elements of a given length $k$ can be parametrized by the Dyck paths of semi-length $n$ with the property that (the sum of peak heights) - (the number of peaks) $=k$ using the canonical form of reduced words for fully commutative elements. For type $D$, from the set of fully commutative element written in canonical form, we decompose the fully commutative elements according to the same type of prefixes and call the set with exactly the same prefix a collection. Then they prove that some collections have the 
same number of elements by showing that those collections contain the same set of prefixes. We then group those collections together and call the group a Packet. This decomposition process is called the packet decomposition.

In the article [25], we generalize the above strategy to the complex reflection group of type $G(d, 1, n)$ for the enumeration of the fully commutative elements of $G(d, 1, n)$. The main result on the combinatorial aspects in Section 5 is that there is a bijection between the standard monomials of $\mathcal{T}(d, n)$ in the first part of this paper and the fully commutative elements of $G(d, 1, n)$ in the second part of this paper, as in Figure 1. In this way, we realize an explicit computation of the dimension of $\mathcal{T}(d, n)$.

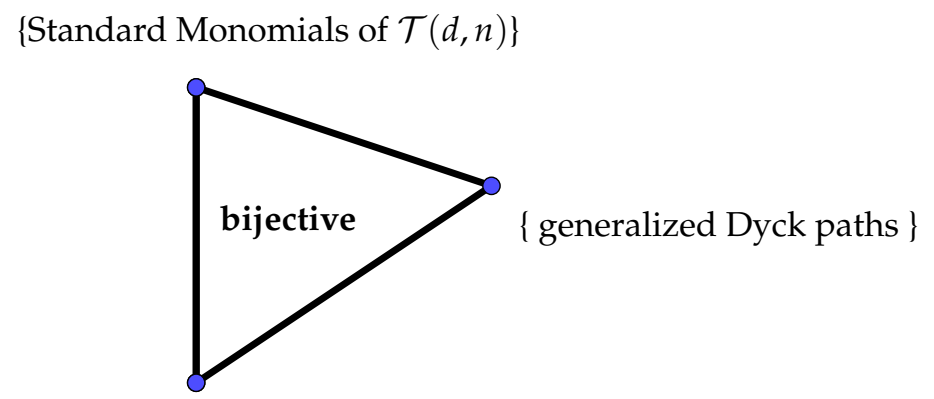

\{fully commutative elements of $G(d, 1, n)$ \}

Figure 1. Three sets are bijective for $G(d, 1, n)$.

Our canonical forms for the reduced elements will be slightly in different form from the ones in [25] and so is the packet decomposition process. Though, the fully commutative elements in this paper are better adapted for the standard monomials induced from a Gröbner-Shirshov basis, having the same number of elements.

\section{Preliminaries}

\section{Gröbner-Shirshov Basis}

We recall a basic theory of Gröbner-Shirshov bases for associative algebras so as to make the paper self-contained. Some properties listed below without proofs are well-known and the readers are invited to see the references cited next to each claim for further detailed explanations.

Let $X$ be a set and let $\langle X\rangle$ be the free monoid of associative words on $X$. We denote the empty word by 1 and the length (or degree) of a word $u$ by $l(u)$. We define a total-order $\langle$ on $\langle X\rangle$, called a monomial order as follows;

$$
\text { if } x<y \text { implies } a x b<a y b \text { for all } a, b \in\langle X\rangle \text {. }
$$

Fix a monomial order $\langle$ on $\langle X\rangle$ and let $\mathbb{F}\langle X\rangle$ be the free associative algebra generated by $X$ over a field $\mathbb{F}$. Given a nonzero element $p \in \mathbb{F}\langle X\rangle$, we denote by $\bar{p}$ the monomial (called the leading monomial) appearing in $p$, which is maximal under the ordering $<$. Thus $p=\alpha \bar{p}+\sum \beta_{i} w_{i}$ with $\alpha, \beta_{i} \in \mathbb{F}$, $w_{i} \in\langle X\rangle, \alpha \neq 0$ and $w_{i}<\bar{p}$ for all $i$. If $\alpha=1, p$ is said to be monic.

Let $S$ be a subset of monic elements in $\mathbb{F}\langle X\rangle$, and let $I$ be the two-sided ideal of $\mathbb{F}\langle X\rangle$ generated by $S$. Then we say that the algebra $A=\mathbb{F}\langle X\rangle / I$ is defined by $S$.

Definition 1. Given a subset $S$ of monic elements in $\mathbb{F}\langle X\rangle$, a monomial $u \in\langle X\rangle$ is said to be $S$-standard (or $S$-reduced) if $u$ cannot be expressed as $a \bar{s} b$, that is $u \neq a \bar{s} b$, for any $s \in S$ and $a, b \in\langle X\rangle$. Otherwise, the monomial $u$ is said to be $S$-reducible.

Lemma 1 ([5,6]). Every $p \in \mathbb{F}\langle X\rangle$ can be expressed as

$$
p=\sum \alpha_{i} a_{i} s_{i} b_{i}+\sum \beta_{j} u_{j},
$$


where $\alpha_{i}, \beta_{j} \in \mathbb{F}, a_{i}, b_{i}, u_{j} \in\langle X\rangle, s_{i} \in S, a_{i} \overline{s_{i}} b_{i} \leq \bar{p}, u_{j} \leq \bar{p}$ and $u_{j}$ are S-standard.

Remark 1. The term $\sum \beta_{j} u_{j}$ in the expression (1) is called a normal form (or a remainder) of $p$ with respect to the subset $S$ (and with respect to the monomial order $<$ ). In general, a normal form is not unique.

As an immediate corollary of Lemma 1, we obtain:

Proposition 1. The set of S-standard monomials spans the algebra $A=\mathbb{F}\langle X\rangle /$ I defined by the subset $S$, as a vector space over $\mathbb{F}$.

Let $p$ and $q$ be monic elements in $\mathbb{F}\langle X\rangle$ with leading monomials $\bar{p}$ and $\bar{q}$. We define the composition of $p$ and $q$ as follows.

Definition 2. (a) If there exist $a$ and $b$ in $\langle X\rangle$ such that $\bar{p} a=b \bar{q}=w$ with $l(\bar{p})>l(b)$, then we define $(p, q)_{w}:=p a-b q$, called the composition of intersection.

(b) If there exist $a$ and $b$ in $\langle X\rangle$ such that $a \neq 1, a \bar{p} b=\bar{q}=w$, then we define $(p, q)_{a, b}:=a p b-q$, called the composition of inclusion.

Let $p, q \in \mathbb{F}\langle X\rangle$ and $w \in\langle X\rangle$. We define the congruence relation on $\mathbb{F}\langle X\rangle$ as follows: $p \equiv q$ $\bmod (S ; w)$ if and only if $p-q=\sum \alpha_{i} a_{i} s_{i} b_{i}$, where $\alpha_{i} \in \mathbb{F}, a_{i}, b_{i} \in\langle X\rangle, s_{i} \in S$, and $a_{i} \overline{s_{i}} b_{i}<w$.

Definition 3. A subset $S$ of monic elements in $\mathbb{F}\langle X\rangle$ is said to be closed under composition if

$(p, q)_{w} \equiv 0 \bmod (S ; w)$ and $(p, q)_{a, b} \equiv 0 \bmod (S ; w)$ for all $p, q \in S, a, b \in\langle X\rangle$ whenever the compositions $(p, q)_{w}$ and $(p, q)_{a, b}$ are defined.

In the subsequent sections, we use the following theorem as a principal method leading us to find a Gröbner-Shirshov basis.

Theorem 2 (Composition Lemma [5,6]). Let $S$ be a subset of monic elements in $\mathbb{F}\langle X\rangle$. Then the following conditions are equivalent:

(a) S is closed under composition.

(b) For each $p \in \mathbb{F}\langle X\rangle$, a normal form of $p$ with respect to $S$ is unique.

(c) The set of $S$-standard monomials forms a linear basis of the algebra $A=\mathbb{F}\langle X\rangle / I$ defined by $S$.

Definition 4. A subset $S$ of monic elements in $\mathbb{F}\langle X\rangle$ satisfying one of the equivalent conditions in Theorem 2 is called a Gröbner-Shirshov basis for the algebra A defined by $S$.

\section{Temperley-Lieb Algebras of Types $A_{n-1}$ and $B_{n}$}

\subsection{Temperley-Lieb Algebra of Type $A_{n-1}$}

First, we review the results on Temperley-Lieb algebras $\mathcal{T}\left(A_{n-1}\right)(n \geq 2)$. Define $\mathcal{T}\left(A_{n-1}\right)$ to be the associative algebra over the complex field $\mathbb{C}$, generated by $X=\left\{E_{1}, E_{2}, \ldots, E_{n-1}\right\}$ with defining relations:

$$
\begin{aligned}
& E_{i}^{2}=\delta E_{i} \quad \text { for } 1 \leq i \leq n-1 \text {, (idempotent relations) } \\
& R_{\mathcal{T}\left(A_{n-1}\right)}: \quad E_{i} E_{j}=E_{j} E_{i} \quad \text { for } i>j+1, \quad \text { (commutative relations) } \\
& E_{i} E_{j} E_{i}=E_{i} \quad \text { for } j=i \pm 1, \quad \text { (untwisting relations) }
\end{aligned}
$$


where $\delta \in \mathbb{C}$ is a parameter. We call the first and second relations the quadratic and commutative relations, respectively. Our monomial order $<$ is taken to be the degree-lexicographic order with

$$
E_{1}<E_{2}<\cdots<E_{n-1} .
$$

We write $E_{i, j}=E_{i} E_{i-1} \cdots E_{j}$ for $i \geq j$ (hence $E_{i, i}=E_{i}$ ). By convention $E_{i, i+1}=1$ for $i \geq 1$.

Proposition 2 ([12], Proposition 6.2). The Temperley-Lieb algebra $\mathcal{T}\left(A_{n-1}\right)$ has a Gröbner-Shirshov basis $\widehat{R}_{\mathcal{T}\left(A_{n-1}\right)}$ as follows:

$$
\begin{array}{rll}
E_{i}^{2}-\delta E_{i} & \text { for } 1 \leq i \leq n-1, \\
\widehat{R}_{\mathcal{T}\left(A_{n-1}\right)}: & E_{i} E_{j}-E_{j} E_{i} & \text { for } i>j+1, \\
E_{i, j} E_{i}-E_{i-2, j} E_{i} & \text { for } i>j, \\
E_{j} E_{i, j}-E_{j} E_{i, j+2} & \text { for } i>j .
\end{array}
$$

The corresponding $\widehat{R}_{\mathcal{T}\left(A_{n-1}\right)}$-standard monomials are of the form

$$
E_{i_{1}, j_{1}} E_{i_{2}, j_{2}} \cdots E_{i_{p}, j_{p}} \quad(0 \leq p \leq n-1)
$$

where

$$
\begin{aligned}
& 1 \leq i_{1}<i_{2}<\cdots<i_{p} \leq n-1, \quad 1 \leq j_{1}<j_{2}<\cdots<j_{p} \leq n-1, \\
& i_{1} \geq j_{1}, i_{2} \geq j_{2}, \ldots, i_{p} \geq j_{p} \quad(\text { the case of } p=0 \text { is the monomial } 1) .
\end{aligned}
$$

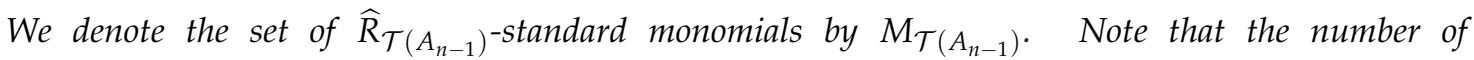
$\widehat{R}_{\mathcal{T}\left(A_{n-1}\right)^{-s t a n d a r d ~ m o n o m i a l s ~ e q u a l s ~ t h e ~} n^{\text {th }} \text { Catalan number, i.e., }}$

$$
\left|M_{\mathcal{T}\left(A_{n-1}\right)}\right|=\frac{1}{n+1}\left(\begin{array}{c}
2 n \\
n
\end{array}\right)=C_{n} .
$$

Remark 2. There are many combinatorial ways to realize the Catalan number $C_{n}$, but among those, it is well-known that $C_{n}$ represents the number of Dyck paths of length $2 n$ (or of semi-length $n$ ) starting from the point $(0,0)$ ending at $(n, n)$ not passing over the diagonal of the $n \times n$-lattice plane.

Example 1. (1) Note that $\left|M_{\mathcal{T}\left(A_{2}\right)}\right|=C_{3}=5$. Explicitly, the $\widehat{R}_{\mathcal{T}\left(A_{2}\right)}$-standard monomials are as follows:

$$
1, E_{1}, E_{2,1}, E_{2}, E_{1} E_{2}
$$

We will give combinatorial interpretations of this set via Dyck paths and fully commutative elements in the last section of this article.

(2) Another example with $n=3$ : we have $\left|M_{\mathcal{T}\left(A_{3}\right)}\right|=C_{4}=14$. Explicitly, the $\widehat{R}_{\mathcal{T}\left(A_{3}\right) \text {-standard }}$ monomials are as follows:

$$
\begin{gathered}
1, E_{1}, E_{2,1}, E_{2}, E_{1} E_{2}, E_{3,1}, E_{3,2}, E_{3} \\
E_{1} E_{3,2}, E_{1} E_{3}, E_{2,1} E_{3,2}, E_{2,1} E_{3}, E_{2} E_{3}, E_{1} E_{2} E_{3} .
\end{gathered}
$$

3.2. Temperley-Lieb Algebra of Type $B_{n}$

Now we consider the Coxeter diagram for type $B_{n}$ :

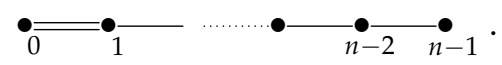

Let $W\left(B_{n}\right)$ be the Weyl group with generators $\left\{s_{i}\right\}_{0 \leq i<n}$ and the following defining relations: 


$$
\begin{aligned}
& \text { - quadratic relations : } s_{i}^{2}=1 \text { for } 0 \leq i \leq n-1 \\
& \text { - braid relations : } \begin{cases}s_{i} s_{j}=s_{j} s_{i} & \text { for }|i-j|>1(i, j=1, \ldots, n), \\
s_{i} s_{i+1} s_{i}=s_{i+1} s_{i} s_{i+1} & \text { for } 1 \leq i<n-1, \\
s_{1} s_{0} s_{1} s_{0}=s_{0} s_{1} s_{0} s_{1} .\end{cases}
\end{aligned}
$$

Then $W\left(B_{n}\right)$ is the Weyl group of type $B_{n}$, which is isomorphic to $(\mathbb{Z} / 2 \mathbb{Z})^{n} \rtimes \mathfrak{S}_{n}$.

Let $\mathcal{H}:=\mathcal{H}\left(B_{n}\right)$ be the Iwahori-Hecke algebra of type $B_{n}$, which is the associative algebra over $A:=\mathbb{Z}\left[q, q^{-1}\right]$, generated by $\left\{T_{i}\right\}_{0 \leq i<n}$ with defining relations:

- quadratic relations: $\left(T_{i}-q\right)\left(T_{i}+q^{-1}\right)=0$ for $0 \leq i \leq n-1$,

$$
\text { - braid relations: } \begin{cases}T_{i} T_{j}=T_{j} T_{i} & \text { for }|i-j|>1(i, j=0, \ldots, n-1), \\ T_{i} T_{i+1} T_{i}=T_{i+1} T_{i} T_{i+1} & \text { for } 1 \leq i<n-1, \\ T_{1} T_{0} T_{1} T_{0}=T_{0} T_{1} T_{0} T_{1} & \end{cases}
$$

where $q$ is a parameter and $T_{i}:=T_{s_{i}}$, the generator corresponding to the reflection $s_{i}$.

Let $\mathcal{T}\left(B_{n}\right)(n \geq 2)$ be the Temperley-Lieb algebra of type $B_{n}$, which is a quotient of the Hecke algebra $\mathcal{H}\left(B_{n}\right) . \mathcal{T}\left(B_{n}\right)$ is the associative algebra over the complex field $\mathbb{C}$, generated by $X=\left\{E_{0}, E_{1}, \ldots, E_{n-1}\right\}$ with defining relations:

$$
\begin{array}{rll}
E_{i}^{2}=\delta E_{i} & \text { for } 0 \leq i \leq n-1, \\
R_{\mathcal{T}\left(B_{n}\right)}: \quad E_{i} E_{j}=E_{j} E_{i} & \text { for } i>j+1, \\
E_{i} E_{j} E_{i}=E_{i} & \text { for } j=i \pm 1, i, j>0, \\
E_{i} E_{j} E_{i} E_{j}=2 E_{i} E_{j} & \text { for }\{i, j\}=\{0,1\},
\end{array}
$$

where $\delta \in \mathbb{C}$ is a parameter. Fix our monomial order $<$ to be the degree-lexicographic order with

$$
E_{0}<E_{1}<\cdots<E_{n-1}
$$

We write $E_{i, j}=E_{i} E_{i-1} \cdots E_{j}$ for $i \geq j \geq 0$, and $E^{i, j}=E_{i} E_{i+1} \cdots E_{j}$ for $i \leq j$. By convention, $E_{i, i+1}=1$ and $E^{i+1, i}=1$ for $i \geq 0$. We can easily prove the following lemma.

Lemma 2 ([16], Section 4). The following relations hold in $\mathcal{T}\left(B_{n}\right)$ :

$$
E_{i, 0} E^{1, j} E_{i}=E_{i-2,0} E^{1, j} E_{i}
$$

for $i>j+1 \geq 1$.

Let $\widehat{R}_{\mathcal{T}\left(B_{n}\right)}$ be the collection of defining relations (7) combined with (2) and the relations in Lemma 2. From this, we denote by $M_{\mathcal{T}\left(B_{n}\right)}$ the set of $\widehat{R}_{\mathcal{T}\left(B_{n}\right)}$-standard monomials. Among the monomials in $M_{\mathcal{T}\left(B_{n}\right)}$, we consider the monomials which are not $\widehat{R}_{\mathcal{T}\left(A_{n-1}\right)}$-standard. That is, we take only $\widehat{R}_{\mathcal{T}\left(B_{n}\right)}$-standard monomials which are not of the form (3). This set is denoted by $M_{\mathcal{T}\left(B_{n}\right)}^{0}$. Note that each monomial in $M_{\mathcal{T}\left(B_{n}\right)}^{0}$ contains $E_{0}$. We decompose the set $M_{\mathcal{T}\left(B_{n}\right)}^{0}$ into two parts as follows:

$$
M_{\mathcal{T}\left(B_{n}\right)}^{0}=M_{\mathcal{T}\left(B_{n}\right)}^{0+} \amalg M_{\mathcal{T}\left(B_{n}\right)}^{0-}
$$


where the monomials in $M_{\mathcal{T}\left(B_{n}\right)}^{0+}$ are of the form

$$
E_{0} E_{i_{1}, j_{1}} E_{i_{2}, j_{2}} \cdots E_{i_{p}, j_{p}} \quad(0 \leq p \leq n-1)
$$

with

$$
\begin{aligned}
& 1 \leq i_{1}<i_{2}<\cdots<i_{p} \leq n-1, \quad 0 \leq j_{1} \leq j_{2} \leq \cdots \leq j_{p} \leq n-1, \\
& i_{1} \geq j_{1}, i_{2} \geq j_{2}, \ldots, i_{p} \geq j_{p}, \text { and } \\
& j_{k}>0(1 \leq k<p) \text { implies } j_{k}<j_{k+1}
\end{aligned}
$$

(the case of $p=0$ is the monomial $E_{0}$ ), and the monomials in $M_{\mathcal{T}\left(B_{n}\right)}^{0-}$ are of the form

$$
E_{i_{1}, j_{1}}^{\prime} E_{i_{2}, j_{2}} \cdots E_{i_{p}, j_{p}} \quad(1 \leq p \leq n-1)
$$

with

$$
E_{i, j}^{\prime}=E_{i, 0} E^{1, j}
$$

and the same restriction on $i^{\prime}$ s and $j$ 's as above. It can be easily checked that $M_{\mathcal{T}\left(B_{n}\right)}^{0}$ is the set of

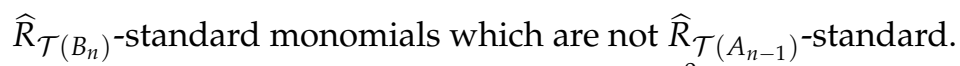

Counting the number of elements in $M_{\mathcal{T}\left(B_{n}\right)}^{0}$, we obtain the following theorem.

Theorem 3 ([16], Section 4). The algebra $\mathcal{T}\left(B_{n}\right)$ has a Gröbner-Shirshov basis $\widehat{R}_{\mathcal{T}\left(B_{n}\right)}$ with respect to our monomial order $<$ :

$$
\begin{aligned}
& E_{i}^{2}-\delta E_{i} \quad \text { for } 0 \leq i \leq n-1, \\
& E_{i} E_{j}-E_{j} E_{i} \quad \text { for } i>j+1 \text {, } \\
& \widehat{R}_{\mathcal{T}\left(B_{n}\right)}: \quad E_{i, j} E_{i}-E_{i-2, j} E_{i} \quad \text { for } i>j>0, \\
& E_{j} E_{i, j}-E_{j} E_{i, j+2} \quad \text { for } i>j>0 \text {. } \\
& E_{i} E_{j} E_{i} E_{j}-2 E_{i} E_{j} \quad \text { for }\{i, j\}=\{0,1\}, \\
& E_{i, 0} E^{1, j} E_{i}-E_{i-2,0} E^{1, j} E_{i} \quad \text { for } i>j+1 \geq 1 \text {. }
\end{aligned}
$$

The cardinality of the set $M_{\mathcal{T}\left(B_{n}\right)}$, i.e., the set of $\widehat{R}_{\mathcal{T}\left(B_{n}\right)}$-standard monomials, is

$$
\operatorname{dim} \mathcal{T}\left(B_{n}\right)=(n+2) C_{n}-1 .
$$

Example 2. For $n=3$, we have $\left|M_{\mathcal{T}\left(B_{3}\right)}\right|=(3+2) C_{3}-1=24$.

We enumerate the standard monomials in $M_{\mathcal{T}\left(B_{3}\right)}^{0}=M_{\mathcal{T}\left(B_{3}\right)} \backslash M_{\mathcal{T}\left(A_{3}\right)}$, that is, the standard monomials containing $E_{0}$, of cardinality $24-5=19$ as follows (using the notation $E_{i, j}^{\prime}:=E_{i, 0} E^{1, j}$ ):

$$
\begin{gathered}
E_{0}, E_{0} E_{1,0}, E_{1,0}, E_{0} E_{1}, E_{1}^{\prime}=E_{1} E_{0} E_{1}, E_{0} E_{2,0}, E_{2,0}, E_{0} E_{2,1}, \\
E_{2,1}^{\prime}=E_{2} E_{1} E_{0} E_{1}, E_{0} E_{2}, E_{2}^{\prime}=E_{2} E_{1} E_{0} E_{1} E_{2}, E_{0} E_{1,0} E_{2,0}, E_{1,0} E_{2,0} \\
E_{0} E_{1,0} E_{2,1}, E_{1,0} E_{2,1}, E_{0} E_{1,0} E_{2}, E_{1,0} E_{2}, E_{0} E_{1} E_{2}, E_{1}^{\prime} E_{2}=E_{1} E_{0} E_{1} E_{2} .
\end{gathered}
$$

Remark 3. As we have seen in the formulas (4) and (8), the dimension of each Temperley-Lieb algebra coincides with the number of fully commutative elements of each corresponding Coxeter group. For this reason, it would be interesting to observe the bijection between the standard monomials for $\mathcal{T}(d, n)$ and the fully commutative elements of $G(d, 1, n)$ in the next section. 


\section{Gröbner-Shirshov Bases for Temperley-Lieb Algebras of the Complex Reflection Group of Type $G(d, 1, n)$}

In this section, we try to generalize the result of the previous section to the complex reflection group of type $G(d, 1, n)$, where $d \geq 2$ and $n \geq 2$. Let $W_{n}:=G(d, 1, n)$ be the wreath product $(\mathbb{Z} / d \mathbb{Z})^{n} \rtimes \mathfrak{S}_{n}$ of the cyclic group $\mathbb{Z} / d \mathbb{Z}$ and the symmetric group $\mathfrak{S}_{n}$, with the following diagram:

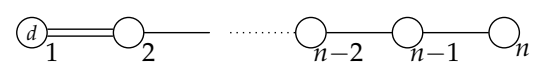

In other words, $W_{n}$ is the group with generators $\left\{s_{i}\right\}_{0 \leq i<n}$ and the following defining relations:

$$
\begin{aligned}
& s_{i}^{2}=1 \text { for } 1 \leq i \leq n-1 \quad \text { and } s_{0}^{d}=1, \\
& \text { braid relations : } \begin{cases}s_{i} s_{j}=s_{j} s_{i} & \text { if }|i-j|>1(i, j=0, \ldots, n-1), \\
s_{i} s_{i+1} s_{i}=s_{i+1} s_{i} s_{i+1} & \text { if } 1 \leq i<n-1, \\
s_{1} s_{0} s_{1} s_{0}=s_{0} s_{1} s_{0} s_{1}\end{cases}
\end{aligned}
$$

Let $\mathcal{H}_{n}:=\mathcal{H}\left(W_{n}\right)$ be the Ariki-Koike algebra of type $G(d, 1, n)$, cyclotomic Hecke algebra which is the associative algebra over $A:=\mathbb{Z}\left[\zeta, q, q^{-1}\right]$, generated by $\left\{T_{i}\right\}_{0 \leq i<n}$ with defining relations:

$$
\begin{aligned}
& \left(T_{i}-q\right)\left(T_{i}+q^{-1}\right)=0 \text { for } 0 \leq i \leq n-1, \\
& \left(T_{0}-q^{m_{0}}\right)\left(T_{0}-q^{m_{1}} \zeta\right)\left(T_{0}-q^{m_{2}} \zeta^{2}\right) \cdots\left(T_{0}-q^{m_{d-1}} \zeta^{d-1}\right)=0, \\
& \text { braid relations : } \begin{cases}T_{i} T_{j}=T_{j} T_{i} & \text { for }|i-j|>1(i, j=0, \ldots, n-1), \\
T_{i} T_{i+1} T_{i}=T_{i+1} T_{i} T_{i+1} & \text { for } 1 \leq i<n-1, \\
T_{1} T_{0} T_{1} T_{0}=T_{0} T_{1} T_{0} T_{1} & \end{cases}
\end{aligned}
$$

where $\zeta$ is a primitive $d^{\text {th }}$ root of unity and $T_{i}:=T_{s_{i}}$, the generator corresponding to the reflection $s_{i}$, and $m_{i} \in \mathbb{N}$.

Similarly, we define the Temperley-Lieb algebra of $G(d, 1, n)(d, n \geq 2)$, denoted by $\mathcal{T}(d, n)$, as a quotient of the Ariki-Koike algebra of $G(d, 1, n)(d, n \geq 2)$.

Definition 5. The Temperley-Lieb algebra $\mathcal{T}(d, n)$ is the associative algebra over the complex field $\mathbb{C}$, generated by $X=\left\{E_{0}, E_{1}, \ldots, E_{n-1}\right\}$ with defining relations:

$$
\begin{array}{cll}
E_{i}^{2}=\delta E_{i} & \text { for } 1 \leq i \leq n-1, \\
R_{\mathcal{T}(d, n)}: & E_{0}^{d}=(d-1) \delta E_{0}, & \\
E_{i} E_{j}=E_{j} E_{i} & \text { for } i>j+1, \\
E_{i} E_{j} E_{i}=E_{i} & \text { for } j=i \pm 1, i, j>0, \\
E_{0} E_{1} E_{0}^{k} E_{1}=(k+1) E_{0} E_{1} & \text { for } 1 \leq k<d, \\
E_{1} E_{0}^{k} E_{1} E_{0}=(k+1) E_{1} E_{0} & \text { for } 1 \leq k<d,
\end{array}
$$

where $\delta \in \mathbb{C}$ is a parameter.

Fix the same monomial order $<$ as in the previous section, i.e., the degree-lexicographic order with $E_{0}<E_{1}<\cdots<E_{n-1}$.

Lemma 3. The following relations hold in $\mathcal{T}(d, n)$;

$$
E_{i, 1} E_{0}^{k} E^{1, j} E_{i}=E_{i-2,1} E_{0}^{k} E^{1, j} E_{i}
$$

for $i>j+1 \geq 1$ and $1 \leq k<d$. 
Proof. Since $2 \leq i \leq n-1$ and $0 \leq j \leq i-2$, we calculate that

$$
E_{i, 1} E_{0}^{k} E^{1, j} E_{i}=\left(E_{i} E_{i-1} E_{i}\right) E_{i-2,1} E_{0}^{k} E^{1, j}=E_{i} E_{i-2,1} E_{0}^{k} E^{1, j}=E_{i-2,1} E_{0}^{k} E^{1, j} E_{i}
$$

by the commutative relations and $E_{i} E_{i-1} E_{i}=E_{i}$.

The set of defining relations (9) combined with (2) and the relations in Lemma 3 is denoted by $\widehat{R}_{\mathcal{T}(d, n)}$. We enumerate $\widehat{R}_{\mathcal{T}(d, n)}$-standard monomials containing $E_{0}$ considering the following two types of standard monomials.

The first type of standard monomials is:

$$
\left(E_{i_{1}, 1} E_{0}^{k_{1}}\right)\left(E_{i_{2}, 1} E_{0}^{k_{2}}\right) \cdots\left(E_{i_{q}, 1} E_{0}^{k_{q}}\right) E_{i_{q+1}, j_{q+1}} \cdots E_{i_{p}, j_{p}} \quad(1 \leq p \leq n-1,1 \leq q \leq p)
$$

with

$$
\begin{aligned}
& 1 \leq k_{1}, k_{2}, \ldots, k_{q}<d, \quad 0 \leq i_{1}<i_{2}<\cdots<i_{q}<i_{q+1}<\cdots<i_{p} \leq n-1, \\
& 1 \leq j_{q+1}<j_{q+2}<\cdots<j_{p} \leq n-1, \quad i_{q+1} \geq j_{q+1}, i_{q+2} \geq j_{q+2}, \ldots, i_{p} \geq j_{p} .
\end{aligned}
$$

In a similar way as we did in the proof of ([16], Theorem 4.2), to count the number of standard monomials of the above form (10), we associate those standard monomials bijectively to the paths defined below.

To each monomial

$$
\left(E_{i_{1}, 1} E_{0}^{k_{1}}\right) \cdots\left(E_{i_{q}, 1} E_{0}^{k_{q}}\right) E_{i_{q+1}, j_{q+1}} \cdots E_{i_{p}, j_{p}}
$$

we associate a unique path, which we call a $G(d, 1, n)$-Dyck path,

$$
\left(i_{1}, 0\right)^{k_{1}} \rightarrow \cdots \rightarrow\left(i_{q}, 0\right)^{k_{q}} \rightarrow\left(i_{q+1}, j_{q+1}\right) \rightarrow \cdots \rightarrow\left(i_{p}, j_{p}\right) \rightarrow(n, n) .
$$

Here, a path consists of moves to the east or to the north, not above the diagonal in the lattice plane. The move from $(i, j)$ to $\left(i^{\prime}, j^{\prime}\right)\left(i<i^{\prime}\right.$ and $\left.j<j^{\prime}\right)$ is a concatenation of eastern moves followed by northern moves. As an example, the monomial $E_{0}^{2} E_{1,0} E_{2,1}$ in $\mathcal{T}(3,3)$ corresponds to the figure below

$$
A(0,0)^{2} \rightarrow B(1,0) \rightarrow C(2,1) \rightarrow(3,3) .
$$

We note that in the move from $B(1,0)$ to $C(2,1)$, there is a concatenation of an eastern move followed by a northern move as well as in the move from $C(2,1)$ to the plot $(3,3)$ as shown in Figure 2 .

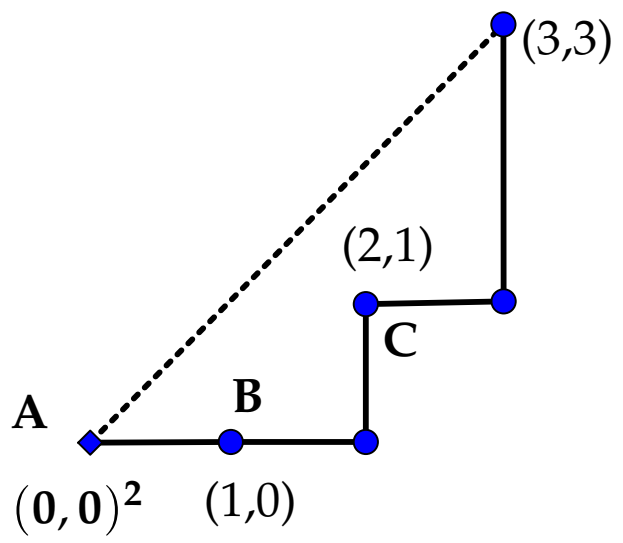

Figure 2. The generalized Dyck path corresponding to $E_{0}^{2} E_{1,0} E_{2,1}$ in $\mathcal{T}(3,3)$.

The second type of standard monomials is:

$$
\left(E_{i_{1}, 1} E_{0}^{k} E^{1, j_{1}}\right) E_{i_{2}, j_{2}} E_{i_{3}, j_{3}} \cdots E_{i_{p}, j_{p}} \quad(1 \leq p \leq n-1)
$$


with

$$
\begin{aligned}
& 1 \leq k<d, \quad 1 \leq i_{1}<i_{2}<\cdots<i_{p} \leq n-1 \\
& 1 \leq j_{1}<j_{2}<\cdots<j_{p} \leq n-1, \quad i_{1} \geq j_{1}, i_{2} \geq j_{2}, \ldots, i_{p} \geq j_{p} .
\end{aligned}
$$

Now we let $M_{\mathcal{T}(d, n)}$ be the set of standard monomials of the forms (10) and (11) combined with (3). The following is our main theorem.

Theorem 4. The algebra $\mathcal{T}(d, n)$ has a Gröbner-Shirshov basis $\widehat{R}_{\mathcal{T}(d, n)}$ with respect to our monomial order $<$ (i.e., degree-lexicographic order with $E_{0}<E_{1}<\cdots<E_{n-1}$ ):

$$
\begin{aligned}
& E_{0}^{d}-(d-1) \delta E_{0} \\
& E_{i} E_{0}-E_{0} E_{i} \quad \text { for } 1<i \leq n-1, \\
& E_{0} E_{1} E_{0}^{k} E_{1}-(k+1) E_{0} E_{1} \quad \text { for } 1 \leq k<d \text {, } \\
& E_{1} E_{0}^{k} E_{1} E_{0}-(k+1) E_{1} E_{0} \quad \text { for } 1 \leq k<d \text {, } \\
& \widehat{R}_{\mathcal{T}(d, n)}: \quad E_{i, 1} E_{0}^{k} E^{1, j} E_{i}-E_{i-2,1} E_{0}^{k} E^{1, j} E_{i} \quad \text { for } i>j+1 \geq 1 \text {, } \\
& E_{i}^{2}-\delta E_{i} \quad \text { for } 1 \leq i \leq n-1, \\
& E_{i} E_{j}-E_{j} E_{i} \quad \text { for } i>j+1>1, \\
& E_{i, j} E_{i}-E_{i-2, j} E_{i} \quad \text { for } i>j>0 \text {, } \\
& E_{j} E_{i, j}-E_{j} E_{i, j+2} \quad \text { for } i>j>0 .
\end{aligned}
$$

The cardinality of the set $M_{\mathcal{T}(d, n)}$, i.e., the set of $\widehat{R}_{\mathcal{T}(d, n)}$-standard monomials, is

$$
\operatorname{dim} \mathcal{T}(d, n)=(d-1)\left(\mathfrak{F}_{n, n-1}(d)-1\right)+d C_{n}
$$

where $\mathfrak{F}_{n, k}(x)=\sum_{s=0}^{k} C(n, s) x^{k-s}$ is the $(n, k)$ th Catalan triangle polynomial, introduced in ([17], Section 2.3).

Proof. Since the set of relations (2) which do not contain $E_{0}$ is already closed under composition, we have only to check for the relations containing $E_{0}$, to show that $\widehat{R}_{\mathcal{T}(d, n)}$ is closed under composition.

- $\quad$ First, consider the compositions between $E_{0}^{d}-(d-1) \delta E_{0}$ and another relation.

$$
\begin{aligned}
- & \left(E_{i} E_{0}-E_{0} E_{i}\right) E_{0}^{d-1}-E_{i}\left(E_{0}^{d}-(d-1) \delta E_{0}\right) \\
& =-E_{0} E_{i} E_{0}^{d-1}+(d-1) \delta E_{i} E_{0}=-E_{i}\left(E_{0}^{d}-(d-1) \delta E_{0}\right)=0 . \\
-\quad & \left(E_{0}^{d}-(d-1) \delta E_{0}\right) E_{1} E_{0}^{k} E_{1}-E_{0}^{d-1}\left(E_{0} E_{1} E_{0}^{k} E_{1}-(k+1) E_{0} E_{1}\right) \\
& =-(d-1) \delta E_{0} E_{1} E_{0}^{k} E_{1}+(k+1) E_{0}^{d} E_{1} \\
& =-(d-1) \delta(k+1) E_{0} E_{1}+(k+1)(d-1) \delta E_{0} E_{1}=0 . \\
- & \left(E_{1} E_{0}^{k} E_{1} E_{0}-(k+1) E_{1} E_{0}\right) E_{0}^{d-1}-E_{1} E_{0}^{k} E_{1}\left(E_{0}^{d}-(d-1) \delta E_{0}\right) \\
& =-(k+1) E_{1} E_{0}^{d}+(d-1) \delta E_{1} E_{0}^{k} E_{1} E_{0} \\
& =-(k+1)(d-1) \delta E_{1} E_{0}+(d-1) \delta(k+1) E_{1} E_{0}=0 .
\end{aligned}
$$

- Next, we take the relation $E_{i} E_{0}-E_{0} E_{i}$ and another relation.

$$
\begin{aligned}
- & \left(E_{i}^{2}-\delta E_{i}\right) E_{0}-E_{i}\left(E_{i} E_{0}-E_{0} E_{i}\right)=-\delta E_{i} E_{0}+E_{i} E_{0} E_{i}=E_{0}\left(-\delta E_{0}+E_{i}^{2}\right)=0 . \\
- & \left(E_{i} E_{0}-E_{0} E_{i}\right) E_{1} E_{0}^{k} E_{1}-E_{i}\left(E_{0} E_{1} E_{0}^{k} E_{1}-(k+1) E_{0} E_{1}\right) \\
& =-E_{0} E_{i} E_{1} E_{0}^{k} E_{1}+(k+1) E_{i} E_{0} E_{1}=-E_{i}\left(E_{0} E_{1} E_{0}^{k} E_{1}-(k+1) E_{0} E_{1}\right)=0 . \\
-\quad & \left(E_{i, 1} E_{0}^{k} E^{1, j} E_{i}-E_{i-2,1} E_{0}^{k} E^{1, j} E_{i}\right) E_{0}-E_{i, 1} E_{0}^{k} E^{1, j}\left(E_{i} E_{0}-E_{0} E_{i}\right) \\
& =-E_{i-2,1} E_{0}^{k} E^{1, j} E_{i} E_{0}+E_{i, 1} E_{0}^{k} E^{1, j} E_{0} E_{i} \\
& =\left(-E_{i-2,1} E_{0}^{k} E^{1, j} E_{i}+E_{i, 1} E_{0}^{k} E^{1, j} E_{i}\right) E_{0}=0 .
\end{aligned}
$$


- $\left(E_{i} E_{j}-E_{j} E_{i}\right) E_{0}-E_{i}\left(E_{j} E_{0}-E_{0} E_{j}\right)=-E_{j} E_{i} E_{0}+E_{i} E_{0} E_{j}=0$.

- $\left(E_{i, j} E_{i}-E_{i-2, j} E_{i}\right) E_{0}-E_{i, j}\left(E_{i} E_{0}-E_{0} E_{i}\right)$

$=-E_{i-2} E_{i} E_{0}+E_{i, j} E_{0} E_{i}=\left(-E_{i-2} E_{i}+E_{i, j} E_{i}\right) E_{0}=0$.

- $\left(E_{j} E_{i, j}-E_{j} E_{i, j+2}\right) E_{0}-E_{j} E_{i, j+1}\left(E_{j} E_{0}-E_{0} E_{j}\right)$

$=-E_{j} E_{i, j+2} E_{0}+E_{j} E_{i, j+1} E_{0} E_{j}=\left(-E_{j} E_{i, j+2}+E_{j} E_{i, j}\right) E_{0}=0$.

- Calculate for the relation $E_{0} E_{1} E_{0}^{k} E_{1}-(k+1) E_{0} E_{1}$ and another relation.

- $\quad\left(E_{0} E_{1} E_{0}^{k} E_{1}-(k+1) E_{0} E_{1}\right) E_{0}^{k} E_{1}-E_{0} E_{1} E_{0}^{k-1}\left(E_{0} E_{1} E_{0}^{k} E_{1}-(k+1) E_{0} E_{1}\right)$ $=-(k+1) E_{0} E_{1} E_{0}^{k} E_{1}+(k+1) E_{0} E_{1} E_{0}^{k} E_{1}=0$.

- $\quad\left(E_{0} E_{1} E_{0}^{k} E_{1}-(k+1) E_{0} E_{1}\right) E_{0}-E_{0}\left(E_{1} E_{0}^{k} E_{1} E_{0}-(k+1) E_{1} E_{0}\right)$ $=-(k+1) E_{0} E_{1} E_{0}+(k+1) E_{0} E_{1} E_{0}=0$.

- $\left(E_{0} E_{1} E_{0}^{k} E_{1}-(k+1) E_{0} E_{1}\right) E_{0}^{k} E_{1} E_{0}-E_{0} E_{1} E_{0}^{k}\left(E_{1} E_{0}^{k} E_{1} E_{0}-(k+1) E_{1} E_{0}\right)$ $=-(k+1) E_{0} E_{1} E_{0}^{k} E_{1} E_{0}+(k+1) E_{0} E_{1} E_{0}^{k} E_{1} E_{0}=0$.

- $\quad\left(E_{1} E_{0}^{k} E_{1} E_{0}-(k+1) E_{1} E_{0}\right) E_{0}^{k-1} E_{1}-E_{1} E_{0}^{k-1}\left(E_{0} E_{1} E_{0}^{k} E_{1}-(k+1) E_{0} E_{1}\right)$ $=-(k+1) E_{1} E_{0}^{k} E_{1}+(k+1) E_{1} E_{0}^{k} E_{1}=0$.

- $\quad\left(E_{1} E_{0}^{k} E_{1} E_{0}-(k+1) E_{1} E_{0}\right) E_{1} E_{0}^{k} E_{1}-E_{1} E_{0}^{k} E_{1}\left(E_{0} E_{1} E_{0}^{k} E_{1}-(k+1) E_{0} E_{1}\right)$ $=-(k+1) E_{1} E_{0} E_{1} E_{0}^{k} E_{1}+(k+1) E_{1} E_{0}^{k} E_{1} E_{0} E_{1}$ $=-(k+1) E_{1}(k+1) E_{0} E_{1}+(k+1)^{2} E_{1} E_{0} E_{1}=0$.

- $\quad\left(E_{0} E_{1} E_{0}^{k} E_{1}-(k+1) E_{0} E_{1}\right) E_{1}-E_{0} E_{1} E_{0}^{k}\left(E_{1}^{2}-\delta E_{1}\right)$ $=-(k+1) E_{0} E_{1}^{2}+\delta E_{0} E_{1} E_{0}^{k} E_{1}=-(k+1) \delta E_{0} E_{1}+\delta(k+1) E_{0} E_{1}=0$.

- $\quad\left(E_{0} E_{1} E_{0}^{k} E_{1}-(k+1) E_{0} E_{1}\right) E_{i, 1}-E_{0} E_{1} E_{0}^{k}\left(E_{1} E_{i, 1}-E_{1} E_{i, 3}\right)$ $=-(k+1) E_{0} E_{1} E_{i, 1}+E_{0} E_{1} E_{0}^{k} E_{1} E_{i, 3}$

$=-(k+1) E_{0} E_{1} E_{i, 1}+(k+1) E_{0} E_{1} E_{i, 3}=-(k+1) E_{0}\left(E_{1} E_{i, 1}-E_{1} E_{i, 3}\right)=0$.

- Check for the relation $E_{1} E_{0}^{k} E_{1} E_{0}-(k+1) E_{1} E_{0}$ and another relation.

- $\quad\left(E_{1} E_{0}^{k} E_{1} E_{0}-(k+1) E_{1} E_{0}\right) E_{0}^{k-1} E_{1} E_{0}-E_{1} E_{0}^{k}\left(E_{1} E_{0}^{k} E_{1} E_{0}-(k+1) E_{1} E_{0}\right)$ $=-(k+1) E_{1} E_{0}^{k} E_{1} E_{0}+(k+1) E_{1} E_{0}^{k} E_{1} E_{0}=0$.

- $\quad\left(E_{1}^{2}-\delta E_{1}\right) E_{0}^{k} E_{1} E_{0}-E_{1}\left(E_{1} E_{0}^{k} E_{1} E_{0}-(k+1) E_{1} E_{0}\right)$ $=-\delta E_{1} E_{0}^{k} E_{1} E_{0}+(k+1) E_{1}^{2} E_{0}=-\delta(k+1) E_{1} E_{0}+(k+1) \delta E_{1} E_{0}=0$.

- $\quad\left(E_{i} E_{1}-E_{1} E_{i}\right) E_{0}^{k} E_{1} E_{0}-E_{i}\left(E_{1} E_{0}^{k} E_{1} E_{0}-(k+1) E_{1} E_{0}\right)$ $=-E_{1} E_{i} E_{0}^{k} E_{1} E_{0}+(k+1) E_{i} E_{1} E_{0}=-E_{i}\left(E_{1} E_{0}^{k} E_{1} E_{0}-(k+1) E_{1} E_{0}\right)=0$.

- $\left(E_{1} E_{i, 1}-E_{1} E_{i, 3}\right) E_{0}^{k} E_{1} E_{0}-E_{1} E_{i, 2}\left(E_{1} E_{0}^{k} E_{1} E_{0}-(k+1) E_{1} E_{0}\right)$ $=-E_{1} E_{i, 3} E_{0}^{k} E_{1} E_{0}+(k+1) E_{1} E_{i, 1} E_{0}=-E_{i, 3}\left(E_{1} E_{0}^{k} E_{1} E_{0}-(k+1) E_{1} E_{0}\right)=0$.

- Finally, consider $E_{i, 1} E_{0}^{k} E^{1, j} E_{i}-E_{i-2,1} E_{0}^{k} E^{1, j} E_{i}$ and another relation.

- $\quad\left(E_{i, 1} E_{0}^{k} E^{1, j} E_{i}-E_{i-2,1} E_{0}^{k} E^{1, j} E_{i}\right) E_{i}-E_{i, 1} E_{0}^{k} E^{1, j}\left(E_{i}^{2}-\delta E_{i}\right)$ $=-E_{i-2,1} E_{0}^{k} E^{1, j} E_{i}^{2}+\delta E_{i, 1} E_{0}^{k} E^{1, j} E_{i}=\delta\left(-E_{i-2,1} E_{0}^{k} E^{1, j} E_{i}+E_{i, 1} E_{0}^{k} E^{1, j} E_{i}\right)=0$.

- $\quad\left(E_{i, 1} E_{0}^{k} E^{1, j} E_{i}-E_{i-2,1} E_{0}^{k} E^{1, j} E_{i}\right) E_{j}-E_{i, 1} E_{0}^{k} E^{1, j}\left(E_{i} E_{j}-E_{j} E_{i}\right)$

$=-E_{i-2,1} E_{0}^{k} E^{1, j} E_{i} E_{j}+E_{i, 1} E_{0}^{k} E^{1, j} E_{j} E_{i}$

$=\left(-E_{i-2,1} E_{0}^{k} E^{1, j} E_{i}+E_{i, 1} E_{0}^{k} E^{1, j} E_{i}\right) E_{j}=0$.

- $\quad\left(E_{i, 1} E_{0}^{k} E^{1, j} E_{i}-E_{i-2,1} E_{0}^{k} E^{1, j} E_{i}\right) E_{i-1, j} E_{i}-E_{i, 1} E_{0}^{k} E^{1, j}\left(E_{i, j} E_{i}-E_{i-2, j} E_{i}\right)$

$=-E_{i-2,1} E_{0}^{k} E^{1, j} E_{i, j} E_{i}+E_{i, 1} E_{0}^{k} E^{1, j} E_{i-2, j} E_{i}$

$=\left(-E_{i-2,1} E_{0}^{k} E^{1, j} E_{i}+E_{i, 1} E_{0}^{k} E^{1, j} E_{i}\right) E_{i-2, j}=0$. 
- $\quad\left(E_{i, 1} E_{0}^{k} E^{1, j} E_{i}-E_{i-2,1} E_{0}^{k} E^{1, j} E_{i}\right) E_{\ell, i}-E_{i, 1} E_{0}^{k} E^{1, j}\left(E_{i} E_{\ell, i}-E_{i} E_{\ell, i+2}\right)$

$=-E_{i-2,1} E_{0}^{k} E^{1, j} E_{i} E_{\ell, i}+E_{i, 1} E_{0}^{k} E^{1, j} E_{i} E_{\ell, i+2}$

$=\left(-E_{i-2,1} E_{0}^{k} E^{1, j} E_{i}+E_{i, 1} E_{0}^{k} E^{1, j} E_{i}\right) E_{\ell, i+2}=0$.

- $\quad\left(E_{j} E_{i, j}-E_{j} E_{i, j+2}\right) E_{j+1,1} E_{0}^{k} E^{1, j} E_{i}-E_{j}\left(E_{i, 1} E_{0}^{k} E^{1, j} E_{i}-E_{i-2,1} E_{0}^{k} E^{1, j} E_{i}\right)$

$=-E_{j} E_{i, j+2} E_{j+1,1} E_{0}^{k} E^{1, j} E_{i}+E_{j} E_{i-2,1} E_{0}^{k} E^{1, j} E_{i}$

$=-E_{j}\left(E_{i, 1} E_{0}^{k} E^{1, j} E_{i}-E_{i-2,1} E_{0}^{k} E^{1, j} E_{i}\right)=0$.

Hence Theorem 2 yields that $\widehat{R}_{\mathcal{T}(d, n)}$ is a Gröbner-Shirshov basis for $\mathcal{T}(d, n)$.

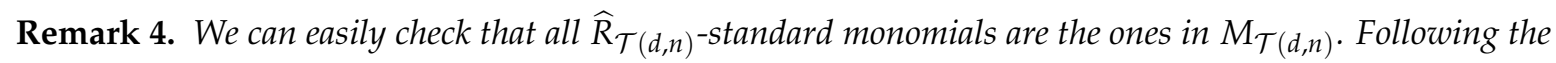
same procedure as in the proof of [16, Theorem 4.2], the number of monomials of the form (10) is

$$
(d-1) \sum_{s=0}^{n-1} C(n, s) d^{n-1-s}=(d-1) \mathfrak{F}_{n, n-1}(d)
$$

by counting the monomials in (10) according to $i_{q}(=n-1-s)=0,1,2, \ldots, n-1$ via $G(d, 1, n)$-Dyck paths.

More precisely, if $i_{q}=0$ then the monomials are of the form $E_{0}^{k} E_{i_{2}, j_{2}} \cdots E_{i_{p}, j_{p}}$, so the number is $(d-1) C_{n}=(d-1) C(n, n-1)$. If $i_{q}=1$ then the number of the monomials is $d(d-1) C(n, n-2)$. If $i_{q}=2$ then the number is $d^{2}(d-1) C(n, n-3)$. In general, if $i_{q}=\ell \leq n-1$ then the number is $d^{\ell}(d-1) C(n, n-1-\ell)$.

On the other hand, the number of monomials of the form (11) is

$$
(d-1)\left(C_{n}-1\right) .
$$

Here, we notice that $p$ in (11) is greater than 0 and thus the identity element is not considered in this case.

Therefore, the cardinality of the set $M_{\mathcal{T}(d, n)}$ is

$$
\begin{aligned}
\operatorname{dim} \mathcal{T}(d, n)=\left|M_{\mathcal{T}(d, n)}\right| & =C_{n}+(d-1) \mathfrak{F}_{n, n-1}(d)+(d-1)\left(C_{n}-1\right) \\
& =(d-1)\left(\mathfrak{F}_{n, n-1}(d)-1\right)+d C_{n} .
\end{aligned}
$$

In particular, with specialisation $d=2$, we recover the result for type $B_{n}$ :

$$
\operatorname{dim} \mathcal{T}\left(B_{n}\right)=(n+2) C_{n}-1
$$

Example 3. Consider the cases of $n=3$. The dimension of $\mathcal{T}(d, 3)$ is

$$
(d-1)\left(\mathfrak{F}_{3,2}(d)-1\right)+d C_{n}=(d-1)\left(d^{2}+3 d+4\right)+5 d=d^{3}+2 d^{2}+6 d-4 .
$$

Notice that, for $n=3$ and $d=3,\left|M_{\mathcal{T}(3,3)} \backslash M_{\mathcal{T}\left(B_{3}\right)}\right|=59-24=35$. The explicit and complete list of the standard monomials in $M_{\mathcal{T}(3,3)} \backslash M_{\mathcal{T}\left(B_{3}\right)}$, that is, the standard monomials containing $E_{0}^{2}$, is as follows:

$$
\begin{gathered}
E_{0}^{2}, E_{0}^{2} E_{1}, E_{0}^{2} E_{1} E_{2}, E_{0}^{2} E_{2,1}, E_{0}^{2} E_{2}, \\
E_{0}^{2} E_{1,0}, E_{0}^{2} E_{1,0} E_{2,1}, E_{0}^{2} E_{1,0} E_{2}, E_{1} E_{0}^{2},\left(E_{1} E_{0}^{2}\right) E_{2,1},\left(E_{1} E_{0}^{2}\right) E_{2}, \\
E_{0}\left(E_{1} E_{0}^{2}\right), E_{0}\left(E_{1} E_{0}^{2}\right) E_{2,1}, E_{0}\left(E_{1} E_{0}^{2}\right) E_{2}, E_{0}^{2}\left(E_{1} E_{0}^{2}\right), E_{0}^{2}\left(E_{1} E_{0}^{2}\right) E_{2,1}, E_{0}^{2}\left(E_{1} E_{0}^{2}\right) E_{2}, \\
E_{0}^{2} E_{2,0}, E_{0}^{2} E_{1,0} E_{2,0},\left(E_{1} E_{0}^{2}\right) E_{2,0}, E_{0}\left(E_{1} E_{0}^{2}\right) E_{2,0}, E_{0}^{2}\left(E_{1} E_{0}^{2}\right) E_{2,0}, \\
E_{2,1} E_{0}^{2}, E_{0}\left(E_{2,1} E_{0}^{2}\right), E_{0}^{2}\left(E_{2,1} E_{0}^{2}\right), E_{1,0}\left(E_{2,1} E_{0}^{2}\right),\left(E_{1} E_{0}^{2}\right)\left(E_{2,1} E_{0}^{2}\right), E_{0} E_{1,0}\left(E_{2,1} E_{0}^{2}\right), \\
E_{0}^{2} E_{1,0}\left(E_{2,1} E_{0}^{2}\right), E_{0}\left(E_{1} E_{0}^{2}\right)\left(E_{2,1} E_{0}^{2}\right), E_{0}^{2}\left(E_{1} E_{0}^{2}\right)\left(E_{2,1} E_{0}^{2}\right), \\
E_{1} E_{0}^{2} E_{1},\left(E_{1} E_{0}^{2} E_{1}\right) E_{2}, E_{2,1} E_{0}^{2} E_{1}, E_{2,1} E_{0}^{2} E^{1,2} .
\end{gathered}
$$




\section{Combinatorial Aspects-Connections to Fully Commutative Elements and Dyck Paths}

In this section, we introduce some combinatorial tools which are useful for the enumeration of standard monomials induced from a Gröbner-Shirshov basis for a Temperley-Lieb algebra of Coxeter groups. We later try to set up a bijective correspondence between the standard monomials and the fully commutative elements.

Let $W$ be a Coxeter group. An element $w \in W$ is said to be fully commutative if any reduced word for $w$ can be obtained from any other by interchanges of adjacent commuting generators. The fully commutative elements play an important role particularly for computing the dimension of the Temperley-Lieb algebra of the Coxeter groups.

Stembridge [22] classified all of the Coxeter groups which have finitely many fully commutative elements. His results completed the work of Fan [21], which was done only for the simply laced types. In the same paper [21], Fan showed that the fully commutative elements parameterize natural bases corresponding to certain quotients of Hecke algebras. In type $A_{n}$, these give rise to the Temperley-Lieb algebras (see [2]). In particular, they showed the following property.

Proposition 3 ([18,21]). Let $C_{n}$ be the nth Catalan number, i.e., $C_{n}=\frac{1}{n+1}\left(\begin{array}{c}2 n \\ n\end{array}\right)$. Then the numbers of fully commutative elements in the Coxeter group of types $A_{n}, D_{n}$ and $B_{n}$ respectively are given as follows:

$$
\begin{cases}C_{n+1} & \text { if the type is } A_{n} \\ \frac{n+3}{2} \times C_{n}-1 & \text { if the type is } D_{n} \\ (n+2) \times C_{n}-1 & \text { if the type is } B_{n} .\end{cases}
$$

Remark 5. There are several combinatorial ways to realize the Catalan number $C_{n}$. Among those, we consider the Dyck paths in $n \times n$-lattice plane starting from the point at $(0,0)$ and ending at the point at $(n, n)$ using only with northern and eastern directions at each step and never passing above the diagonal line. Seeing that the dimension of the Temperley-Lieb algebra $\mathcal{T}\left(A_{n-1}\right)$ of the Coxeter group of type $A_{n-1}$ is the Catalan number $C_{n}$ which is the number of fully commutative elements, we can realize a bijective correspondence between the Dyck paths and the fully commutative elements for $\mathcal{T}\left(A_{n-1}\right)$ as we can see in the article [24].

The number of fully commutative elements in Coxeter groups of type $B$ and $D$ respectively in Proposition 3 above can also be understood in pure combinatorial way by using the notions of Catalan's triangle and Packets as mentioned in the articles [23,25]. In this section, we recall the definitions and some combinatorial properties of Catalan numbers and Catalan triangles as well as the main results in $[23,25]$.

The Catalan numbers can be defined in a recursive way as follows: we set the first entry $C(0,0)=1$. For $n \geq 0$ and $0 \leq k \leq n$, we denote $C(n, k)(0 \leq k \leq n)$ the entry in the $n$th row and $k$ th column of Table ?? below, called Catalan's Triangle, verifying the following recursive formula.

$$
C(n, k)=\left\{\begin{array}{cl}
1 & \text { if } n=0 ; \\
C(n, k-1)+C(n-1, k) & \text { if } 0<k<n ; \\
C(n-1,0) & \text { if } k=0 ; \\
C(n, n-1)=C(n, n)=C_{n} & \text { if } k=n . \\
0 & \text { if } k>n .
\end{array}\right.
$$

Remark 6. We consider the Dyck paths in $n \times n$-lattice plane starting from the point at $(0,0)$ and ending at the point at $(n, n)$ using only with northern and eastern directions at each step and never passing above the diagonal line. Then the coefficient $C(n, k)$ in the Catalan's triangle can be understood as the number of these Dyck paths passing through exactly the point $(n, k)(k \leq n)$.

In [23], Feinberg and Lee showed that the set of fully commutative elements can be decomposed into subsets called collections depending on the shape of the suffix of each reduced element written in 
canonical form. Then they proved that some collections have the same set of prefixes, i.e., the same cardinality. Now, we group those collections together and call the group a packet and we call this process the packet decomposition.

Table 1. Catalan's Triangle.

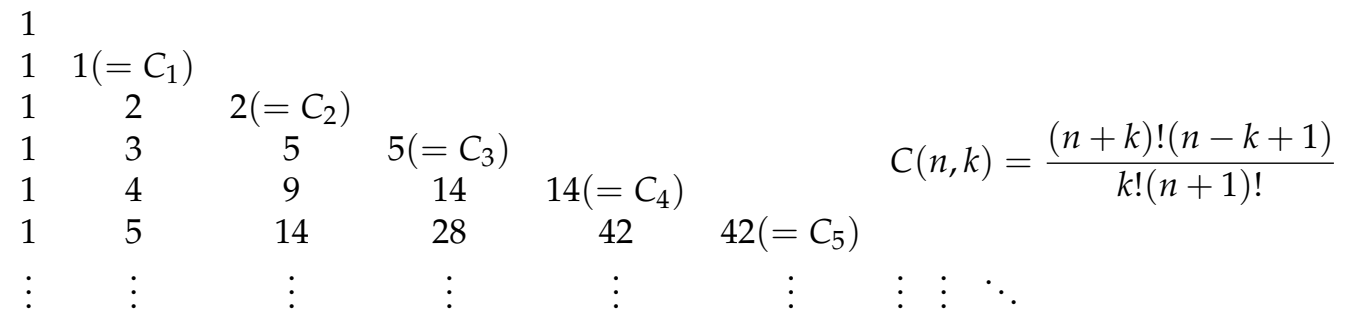

Following the notations in (5) and (6), for $0 \leq i<n$, we define the words $s_{i j}$ and $s^{j, i}$ by:

$$
s_{i j}=\left\{\begin{array}{ll}
{[i, i-1, \ldots, j]:=s_{i} \cdots s_{j}} & \text { if } i>j, \\
{[i]:=s_{i}} & \text { if } i=j, \\
{[]:=1} & \text { if } i<j .
\end{array} \quad s^{j i}= \begin{cases}s_{j} s_{j+1} \cdots s_{i} & \text { if } i>j, \\
{[i]:=s_{i}} & \text { if } i=j, \\
{[]:=1} & \text { if } i<j .\end{cases}\right.
$$

Lemma 4 ([13], Proposition 2.3, cf. [7], Lemma 4.2). Any element of the Coxeter group of type $B_{n}$ can be uniquely written in the reduced form

$$
s_{0, a_{0}}^{\left(k_{0}\right)} s_{1, a_{1}}^{\left(k_{1}\right)} s_{2, a_{2}}^{\left(k_{2}\right)} \cdots s_{n-1, a_{n-1}}^{\left(k_{n-1}\right)}
$$

where

$$
1 \leq a_{i} \leq i+1, k_{i}=0 \text { or } 1, \text { and } s_{i j}^{(k)}= \begin{cases}s_{i j} & \text { if } k=0, \\ s_{i, 0} s^{1, j-1} & \text { if } k=1 .\end{cases}
$$

Remark 7. Notice that there are $i+1$ choices for each $a_{i}$ since $1 \leq a_{i} \leq i+1$ while $i=0,1, \ldots, n-1$, thus $n$ ! choices for the values of $a_{i}$ 's altogether. There are 2 ways for each exponent $k_{i}$ of $a_{i}$, and thus there are $2^{n}$ choices for the exponents. Thus we have $n ! \cdot 2^{n}$ elements in the canonical reduced form. We recall that there are the same number of elements in the type $B_{n}$ Coxeter group.

Among the above canonical elements, since, for $1 \leq i_{1}<i_{2}$ and $j_{1}>1$, any element

$$
s_{i_{1}, j_{1}}^{(1)} s_{i_{2}, j_{2}}^{(1)}=s_{i_{1}, 0} s^{1, j_{1}-1} s_{i_{2}, o^{1, j_{2}}} s^{1}=s_{i_{1}, 0} s^{1, j_{1}-2} s_{i_{2}, j_{1}+1}\left(s_{j_{1}-1} s_{j_{1}} s_{j_{1}-1}\right) s_{j_{1}-2,0} s^{1, j_{2}-1}
$$

contains a braid relation $s_{j-1} s_{j} s_{j-1}$, thus any element containing this form is not fully commutative. Also, for $j>1$, any element containing the form

$$
s_{0} s_{i, j}^{(1)}=s_{0} s_{i, 0} s^{1, j-1}=s_{i, 2}\left(s_{0} s_{1} s_{0} s_{1}\right) s^{2, j-1}
$$

is a non-fully commutative element.

Now we consider only the elements of the form

$$
s_{i_{1}, 0} s_{i_{2}, 0} \cdots s_{i_{\ell}, 0} s_{i_{\ell}+1, a_{i_{\ell}+1}} \cdots s_{n-1, a_{n-1}}
$$

where $0 \leq i_{1}<i_{2}<\cdots<i_{\ell}<n$ for $\ell \geq 0$, or

$$
\left(s_{i, 0} s^{1, a_{i}}\right) s_{i+1, a_{i+1}} \cdots s_{n-1, a_{n-1}}
$$

with $i \geq 1$. 
The left factors $s_{i_{1}, 0} s_{i_{2}, 0} \cdots s_{i_{\ell}, 0}$ in (12), and $s_{i, 0}$ in (13) will be called the prefix. Similarly the right factors $s_{i_{\ell}+1, a_{i}+1} \cdots s_{n-1, a_{n-1}}$ in (12), and $s^{1, a_{i}} s_{i+1, a_{i+1}} \cdots s_{n-1, a_{n-1}}$ in (13) will be called the suffix of the reduced word. Given a reduced word $w$ in the extracted canonical form, we will denote by $w_{p}$ the prefix and by $\boldsymbol{w}_{s}$ the suffix of $\boldsymbol{w}$ and we can write, in a unique way, $\boldsymbol{w}=\boldsymbol{w}_{p} \boldsymbol{w}_{s}$.

We notice that every prefix is a fully commutative element. Some of the collections in the set of fully commutative elements of $W\left(B_{n}\right)$ have the same number of elements and thus we group them together into a set called a $(n, k)$-packet depending on the form of the prefixes as follows:

Definition 6. For $0 \leq k \leq n$, we define the $(n, k)$-packet of collections:

- The $(n, 0)$-packet is the set of collections labeled by prefixes of the form

$$
s_{i_{1}, 0} s_{i_{2}, 0} \cdots s_{i_{\ell}, 0} s_{n-1,0} \quad(\ell \geq 1) .
$$

- $\quad$ The $(n, k)$-packet, $1 \leq k \leq n-2$, is the set of collections labeled by $s_{n-k, 0}$ or prefixes of the form

$$
s_{i_{1}, 0} s_{i_{2}, 0} \cdots s_{i_{\ell}, 0} s_{n-k-1,0} \quad(\ell \geq 1) .
$$

- The $(n, n-1)$-packet contains only the collection labeled by $s_{0}=[0]$ or $s_{1,0}=[1,0]$.

- The $(n, n)$-packet contains only the collection labeled by the empty prefix [ ].

We will denote the $(n, k)$-packet by $\mathcal{P}_{B}(n, k)$. As an example, Table 2 shows all of collections of the packets for type $B_{3}$.

Proposition 4 ([25]). The cardinality of the packet $\mathcal{P}_{B}(n, k)$ for type $B_{n}$ is

$$
\left|\mathcal{P}_{B}(n, k)\right|=\left\{\begin{array}{cl}
2^{n-1}-1 & \text { if } k=0, \\
2^{n-k-1} & \text { if } 1 \leq k \leq n-2, \\
2 & \text { if } k=n-1, \\
1 & \text { if } k=n .
\end{array}\right.
$$

Therefore $\sum_{k=0}^{n}\left|\mathcal{P}_{B}(n, k)\right|=2^{n}$. We remark that for a fixed $k$ with $0 \leq k \leq n((n \geq 3)$, each collection with same prefix in the packet $\mathcal{P}_{B}(n, k)$ has the same cardinality which is $C(n, k)$.

Corollary 1. For $n \geq 3$, we obtain the identity:

$$
\sum_{k=0}^{n} C(n, k)\left|\mathcal{P}_{B}(n, k)\right|=(n+2) \times C_{n}-1 .
$$

We generalize the above method for the complex reflection group of type $G(d, 1, n)$.

Lemma 5 (Canonical Form for $G(d, 1, n)$ [13], Proposition 2.3). Any element of the Coxeter group of type $G(d, 1, n)$ can be uniquely written in the reduced form

$$
s_{0, a_{0}}^{\left(k_{0}\right)} s_{1, a_{1}}^{\left(k_{1}\right)} s_{2, a_{2}}^{\left(k_{2}\right)} \cdots s_{n-1, a_{n-1}}^{\left(k_{n-1}\right)}
$$

where

$$
1 \leq a_{i} \leq i+1,0 \leq k_{i} \leq d-1, \text { and } s_{i j}^{(k)}= \begin{cases}s_{i j} & \text { if } k=0, \\ s_{i, 1} s_{0}^{k} s^{1, j-1} & \text { if } k=1,2, \ldots, d-1 .\end{cases}
$$

Remark 8. Notice that there are $n !$ choices of $a_{i}$ 's $(i=0,1, \ldots, n-1)$, and there are $d^{n}$ ways to choose $k_{i}$ 's. Therefore we have $n ! \cdot d^{n}$ elements in the canonical reduced form. We recall that there are the same number of elements in the complex reflection group of type $G(d, 1, n)$. 
The fully commutative elements of the complex reflection group of type $G(d, 1, n)$ are of the form

$$
s_{i_{1}, 1} s_{0}^{k_{1}} s_{i_{2}, 1} s_{0}^{k_{2}} \cdots s_{i_{q}, 1} s_{0}^{k_{q}} s_{i_{q+1}, j_{q+1}} \cdots s_{i_{p}, j_{p}}
$$

with

$$
\begin{aligned}
& 0 \leq p \leq n, \quad 0 \leq q \leq p, \quad 1 \leq k_{1}, k_{2}, \ldots, k_{q}<d \\
& 0 \leq i_{1}<i_{2}<\cdots<i_{p} \leq n-1, \quad 0<j_{q+1}<\cdots \leq j_{p} \leq n-1, \text { and } \\
& i_{q+1} \geq j_{q+1}, \ldots, i_{p} \geq j_{p},
\end{aligned}
$$

or

$$
\left(s_{i_{1}, 1} s_{0}^{k} s^{1, j_{1}}\right) s_{i_{2}, j_{2}} s_{i_{3}, j_{3}} \cdots s_{i_{p}, j_{p}}
$$

with

$$
\begin{aligned}
& 1 \leq p \leq n-1, \quad 1 \leq k<d \\
& 1 \leq i_{1}<i_{2}<i_{3}<\cdots<i_{p} \leq n-1, \quad 1 \leq j_{1}<j_{2}<j_{3}<\cdots<j_{p} \leq n-1, \text { and } \\
& i_{1} \geq j_{1}, i_{2} \geq j_{2}, \ldots, i_{p} \geq j_{p} .
\end{aligned}
$$

The left factor $s_{i_{1}, 1} s_{0}^{k_{1}} s_{i_{2}, 1} s_{0}^{k_{2}} \cdots s_{i_{q}, 1} s_{0}^{k_{q}}$ with $q \geq 0$ in (15) and (16) will be called the prefix of the fully commutative element of type $G(d, 1, n)$.

Definition 7. For $0 \leq s \leq n$, we define the $(n, s)$-packet of collections:

- The $(n, 0)$-packet is the set of collections labeled by prefixes of the form

$$
s_{i_{1}, 1} s_{0}^{k_{1}} s_{i_{2}, 1} s_{0}^{k_{2}} \cdots s_{i_{q-1}, 1} s_{0}^{k_{q-1}} s_{n-1,1} s_{0}^{k_{q}} \quad(q \geq 2) .
$$

- The $(n, s)$-packet, $1 \leq s \leq n-2$, is the set of collections labeled by $s_{n-s, 1} s_{0}^{k}$ or prefixes of the form

$$
s_{i_{1}, 1} s_{0}^{k_{1}} s_{i_{2}, 1} s_{0}^{k_{2}} \cdots s_{i_{q-1}, 1}^{k_{q-1}} s_{n-s-1,1} s_{0}^{k_{q}} \quad(q \geq 2) .
$$

- The $(n, n-1)$-packet contains only the collections labeled by $s_{0}^{k}=\left[0^{k}\right]$ or $s_{1} s_{0}^{k}=\left[1,0^{k}\right]$.

- The $(n, n)$-packet contains only the collection labeled by the empty prefix []. These elements cover the elements of $\mathfrak{S}_{n} \subset G(d, 1, n)$.

We will denote the $(n, s)$-packet by $\mathcal{P}(n, s)$. For clarifying the packet decomposition, we denote $(n, s)$-packet of type $G(d, 1, n)$ which is not contained in the $(n, s)$-packet of type $B_{n}$ by

$$
\mathcal{P}(n, s)^{\prime}=\mathcal{P}(n, s) \backslash \mathcal{P}_{B}(n, s) .
$$

Proposition 5 (cf. [25], Section 4).

- Every collection in the packet $\mathcal{P}(n, s)$ has $C(n, s)$ elements.

- $\quad$ The size of the packet $\mathcal{P}(n, s)$ is

$$
|\mathcal{P}(n, s)|= \begin{cases}(d-1)\left(d^{n-1}-1\right) & \text { if } s=0, \\ (d-1) d^{n-1-s} & \text { if } 1 \leq s \leq n-2, \\ 2(d-1) & \text { if } s=n-1 \\ 1 & \text { if } s=n .\end{cases}
$$

Proof. (a) The collections in $\mathcal{P}(n, 0)$ have no other element than the prefix. It is also clear that the collections in $\mathcal{P}(n, n-1)$ have $C_{n}=C(n, n-1)$ elements and the collection in $\mathcal{P}(n, n)$ has $C_{n}=C(n, n)$ elements. 
For the collections in $\mathcal{P}(n, s)$ with $1 \leq s \leq n-2$, the number of suffixes attached to the given prefix is exactly $C(n, s)$ by counting the Dyck paths from $(n-s, 0)$ to $(n, n)$ via the Dyck paths, reflected relative to $y=n-x$ in the $x y$-plane, from $(0,0)$ to $(n, s)$.

(b) Counting the number of prefixes in each packet, we get its packet size.

In the same way as the formula (14), we obtain the identity for the complex reflection group of type $G(d, 1, n)$ :

$$
\begin{aligned}
\sum_{s=0}^{n} C(n, s)|\mathcal{P}(n, s)| & =(d-1)\left(d^{n-1}-1\right)+\sum_{s=1}^{n-2} C(n, s)(d-1) d^{n-1-s}+2(d-1) C_{n}+C_{n} \\
& =(d-1)\left(\mathfrak{F}_{n, n-1}(d)-1\right)+d C_{n},
\end{aligned}
$$

which is equal to $\operatorname{dim} \mathcal{T}(d, n)$.

As an example, Table 2 shows all of collections of the packets for type $G(3,1,3)$.

Table 2. Bijection between Standard Monomials and Fully Commutative Elements of $G(3,1,3)$.

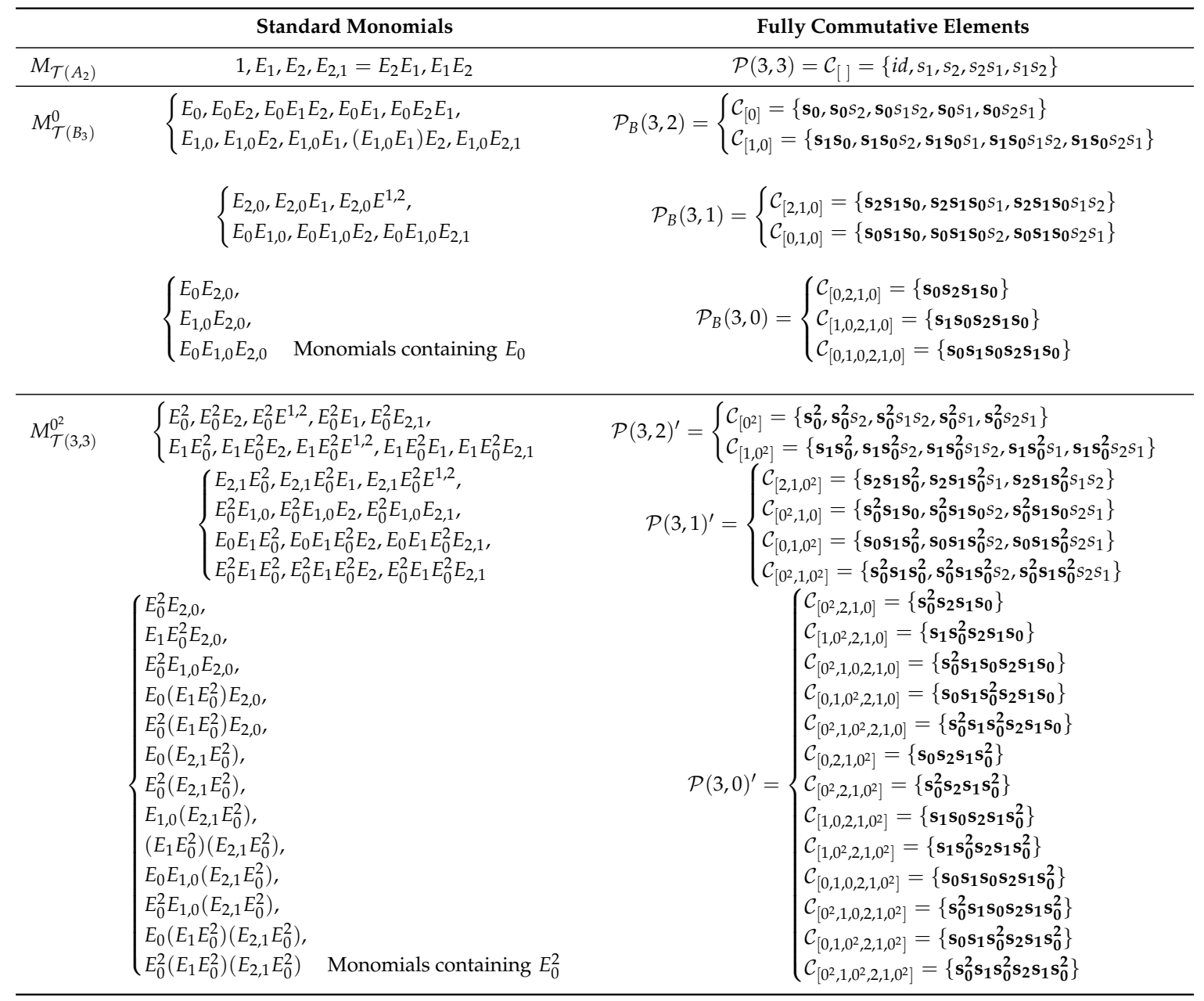

\section{Temperley-Lieb Algebras of Types $G(d, d, n)$ and $G(d, r, n)$}

From a $\mathbb{C}$-basis of $\mathcal{T}(d, n)$ and its multiplication structure, we define the subalgebras $\mathcal{T}(d, d, n)$ and $\mathcal{T}(d, r, n)$ in general as follows. 


\section{Definition 8.}

- $\quad$ The subalgebra $\mathcal{T}(d, d, n)$ is a subalgebra of $\mathcal{T}(d, n)$, whose $\mathbb{C}$-basis consists of

$$
\left\{\begin{array}{l}
\text { the elements in (3), } \\
\text { the elements in (10) satisfying } k_{1}+\cdots+k_{q} \equiv 0 \quad(\bmod d), \\
\text { the elements in (11) with } k=1 .
\end{array}\right.
$$

We remark that

$$
\begin{aligned}
\operatorname{dim} \mathcal{T}(d, d, n) & =C_{n}+(d-1) \mathfrak{F}_{n, n-2}(d)+\left(C_{n}-1\right) \\
& =(d-1) \mathfrak{F}_{n, n-2}(d)+2 C_{n}-1
\end{aligned}
$$

In particular, for $d=2$, we recover the same formula as we had in [16]:

$$
\operatorname{dim} \mathcal{T}(2,2, n)=\operatorname{dim} \mathcal{T}\left(D_{n}\right)=\frac{n+3}{2} C_{n}-1
$$

- More generally, the subalgebra $\mathcal{T}(d, r, n)(r \mid d$ and $r \geq 2)$ is a subalgebra of $\mathcal{T}(d, n)$, whose $\mathbb{C}$-basis consists of

$$
\left\{\begin{array}{l}
\text { the elements in (3), } \\
\text { the elements in (10) satisfying } k_{1}+\cdots+k_{q} \equiv 0 \quad(\bmod r), \\
\text { the elements in (11) with } k \in\{1, r, 2 r, \cdots, d-2 r, d-r\} .
\end{array}\right.
$$

We remark that

$$
\begin{aligned}
\operatorname{dim} \mathcal{T}(d, r, n) & =C_{n}+\frac{d}{r}(d-1) \mathfrak{F}_{n, n-2}(d)+\frac{d}{r}\left(C_{n}-1\right) \\
& =\frac{d}{r}(d-1) \mathfrak{F}_{n, n-2}(d)+\left(1+\frac{d}{r}\right) C_{n}-\frac{d}{r}
\end{aligned}
$$

Remark 9. The set of the above $\mathbb{C}$-basis monomials of $\mathcal{T}(d, d, n)$ and $\mathcal{T}(d, r, n)$, respectively, is closed under multiplication with the same arguments as we had for $\mathcal{T}(d, n)$.

Example 4. The standard monomials for $\mathcal{T}(3,3,3)$ are as follows:

$$
\begin{gathered}
1, E_{1}, E_{2}, E_{2,1}, E_{1} E_{2}, \\
E_{0} E_{1,0} E_{2,0}, E_{0}^{2} E_{1,0}, E_{0}^{2} E_{1,0} E_{2}, E_{0}^{2} E_{1,0} E_{2,1}, E_{0} E_{1} E_{0}^{2}, E_{0} E_{1} E_{0}^{2} E_{2}, E_{0} E_{1} E_{0}^{2} E_{2,1}, \\
E_{0}^{2} E_{2,0}, E_{1} E_{0}^{2} E_{2,0}, E_{0}\left(E_{2,1} E_{0}^{2}\right), E_{1,0}\left(E_{2,1} E_{0}^{2}\right), E_{0}^{2}\left(E_{1} E_{0}^{2}\right)\left(E_{2,1} E_{0}^{2}\right), \\
E_{1,0} E_{1},\left(E_{1,0} E_{1}\right) E_{2}, E_{2,0} E_{1}, E_{2,0} E^{1,2} .
\end{gathered}
$$

Thus we obtain that

$$
\operatorname{dim} \mathcal{T}(3,3,3)=C_{3}+(3-1) \mathfrak{F}_{3,1}(3)+\left(C_{3}-1\right)=5+12+4=21 .
$$

Author Contributions: The authors contributed equally to this paper.

Funding: The first author's research was supported by NRF Grant \# 2017078374. The research of the corresponding author was supported by NRF Grant \# 2018R1D1A1B07044111 and a research grant from Seoul Women's University(2018).

Acknowledgments: S. Kim is grateful to KIAS-CMC for its hospitality during her visit. The authors are grateful to the reviewers for their suggestions.

Conflicts of Interest: The authors declare no conflicts of interest. 


\section{References}

1. Temperley, H.N.V.; Lieb, E.H. Relations between percolation and colouring problems and other graph theoretical problems associated with regular planar lattices: Some exact results for the percolation problem. Proc. R. Soc. Lond. Ser. A 1971, 322, 251-280. [CrossRef]

2. Jones, V.F.R. Hecke algebra representations of braid groups and link polynomials. Ann. Math. 1987, 126, 335-388. [CrossRef]

3. Shirshov, A.I. Some algorithmic problems for Lie algebras. Sibirk. Math. Z. 1962, 3, 292-296 (In Russian). Translated in ACM SIGSAM Bull. Commun. Comput. Algebra 1999, 33, 3-6.

4. Buchberger, B. An Algorithm for Finding the Basis Elements of the Residue Class Ring of a Zero Dimensional Polynomial Ideal. Ph.D. Thesis, University of Innsbruck, Innsbruck, Austria, 1965 (In German). Translated in J. Symb. Comput. 2006, 41, 475-511.

5. Bokut, L.A. Imbedding into simple associative algebras. Algebra Logic 1976, 15, 117-142. [CrossRef]

6. Bergman, G.M. The diamond lemma for ring theory. Adv. Math. 1978, 29, 178-218. [CrossRef]

7. Bokut, L.A.; Shiao, L.-S. Gröbner-Shirshov bases for Coxeter groups. Comm. Algebra 2001, 29, 4305-4319. [CrossRef]

8. Lee, D. Gröbner-Shirshov bases and normal forms for the Coxeter groups $E_{6}$ and $E_{7}$. In Advances in Algebra and Combinatorics; World Scientific Pub.: Singapore, 2008; pp. 243-255.

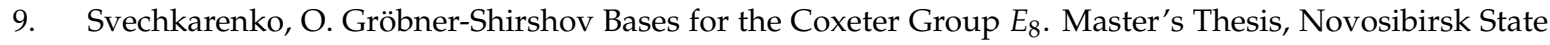
University, Novosibirsk, Russia, 2007.

10. Lee, D.-I. Standard monomials for the Weyl group F. J. Algebra Appl. 2016, 15, 1650146. [CrossRef]

11. Lee, D.-I.; Lee, J.-Y. Gröbner-Shirshov bases for non-crystallographic Coxeter groups. arXiv 2018, arXiv:1809.08419.

12. Kang, S.-J.; Lee, I.-S.; Lee, K.-H.; Oh, H. Hecke algebras, Specht modules and Gröbner-Shirshov bases. J. Algebra 2002, 252, 258-292. [CrossRef]

13. Kang, S.-J.; Lee, I.-S.; Lee, K.-H.; Oh, H. Representations of Ariki-Koike algebras and Gröbner-Shirshov bases. Proc. Lond. Math. Soc. 2004, 89, 54-70. [CrossRef]

14. Lee, D.-I. Cyclotomic Hecke algebras of $G(r, p, n)$. Algebr. Represent. Theory 2010, 13, 705-718. [CrossRef]

15. Dattoil, G.; Ricci, P.E.; Cesarano, C. Beyond the monomiality: The monumbrality principle. J. Comput. Anal. Appl. 2004, 6, 77-83.

16. Kim, S.; Lee, D.-I. Gröbner-Shirshov bases for Temperley-Lieb algebras of types B and D. arXiv 2017, arXiv:1808.05026.

17. Lee, K.-H.; Oh, S.-J. Catalan triangle numbers and binomial coefficients. Contemp. Math. 2018, 713, $165-185$.

18. Stembridge, J.R. Some combinatorial aspects of reduced words in finite Coxeter groups. Trans. Am. Math. Soc. 1997, 349, 1285-1332. [CrossRef]

19. Kleshchev, A.; Ram, A. Homogeneous representations of Khovanov-Lauda algebras. J. Eur. Math. Soc. 2010, 12, 1293-1306. [CrossRef]

20. Kleshchev, A.; Ram, A. Representations of Khovanov-Lauda-Rouquier algebras and combinatorics of Lyndon words. Math. Ann. 2011, 349, 943-975. [CrossRef]

21. Fan, C.K. A Hecke Algebra quotient and some combinatorial applications. J. Algebraic Combin. 1996, 5, 175-189. [CrossRef]

22. Stembridge, J.R. On the fully commutative elements of Coxeter groups. J. Algebraic Combin. 1996, 5, 353-385. [CrossRef]

23. Feinberg, G.; Lee, K.-H. Fully commutative elements of type $D$ and homogeneous representations of KLR-algebras. J. Comb. 2015, 6, 535-557. [CrossRef]

24. Feinberg, G.; Lee, K.-H. Homogeneous representations of type A KLR-algebras and Dyck paths. Sém. Lothar. Combin. 2016, 75, Art. B75b.

25. Feinberg, G.; Kim, S.; Lee, K.-H.; Oh, S.-J. Fully commutative elements of the complex reflection groups. arXiv 2018, arXiv:1808.04269. 